\title{
LOSS OF PRIMARY MOLARS SPACE (D E SPACE) DUE TO UNTREATED INTERPROXIMAL CARIES: A CROSS-SECTIONAL STUDY
}

\author{
Amr E. Abdel Latif*
}

\begin{abstract}
Aim: to assess the changes taking place in D E space caused by untreated interproximal caries of primary molars during the mixed dentition.

Subjects and methods: This study was a cross-sectional one. A sample of 52 children between 7 to 9 years of age with unilateral interproximal caries of first and/or second primary mandibular molars was assessed to detect the change in D E space caused by loss of contacts due to interproximal caries. D E space change was defined as the change in the distance between the midpoint on the mesial surface of the mandibular permanent first molar and the midpoint on the distal surface of the mandibular primary canine (DE Space). The space change between damaged sides and the intact control sides was measured by cast analysis using digital caliper. Interexaminer reliability was high (0.970).
\end{abstract}

Results: The results showed the measured change of D E space on the damaged sides was significantly reduced when compared to the control sides $(\mathrm{P}<0.001)$.

Conclusion: The study concluded that the space needed for eruption of permanent teeth decrease significantly after loss of proximal contacts of primary molars due to interproximal caries in mixed dentition stage.

\section{INTRODUCTION}

The primary dentition plays a very important role in the child's growth and development, not only in terms of speech, chewing, appearance and the prevention of bad habits but also in the guidance and eruption of permanent teeth. Exfoliation of primary teeth and eruption of permanent teeth is a normal physiological process. When this normal process is disturbed, due to factors like premature loss of primary teeth, proximal carious lesions etc, it may lead to mesial migration of teeth resulting in loss of the arch length which may manifest as malocclusion in permanent dentition in the form of crowding, impaction of permanent teeth, super eruption of opposing teeth etc. ${ }^{[1-3]}$.

* Associate Professor Pediatric Dentistry and Dental Public Health, Faculty of Dentistry, Cairo University 
One of the important areas involved in the prevention of dental disease in children is that of maintaining (as far as is clinically possible) a normal growth pattern of the face and dental arches during the periods of the primary dentition and of the "mixed dentition," i.e. that period during which these primary teeth are being shed and replaced by their permanent successors. One of the important services that the general dentist and the pedodontist can render for young patient is that of maintaining the arch length prior to the eruption of permanent teeth ${ }^{[4]}$

Previous studies by Seward et.al and Northway et al. ${ }^{[5,6]}$ blamed caries to be the important factor in development of malocclusion, both agreed that interproximal caries of deciduous dentition results narrowing of the required space for eruption of permanent successors.

The concept described in the present study has been tailored to portray the volatile changes occurring in the buccal segment caused by untreated interproximal caries of primary molars during the mixed dentition. It has been named D E Space and has been defined as the distance between the midpoint on the mesial surface of the permanent first molar and the midpoint on the distal surface of the primary canine ${ }^{[7]}$

\section{SUBJECTS AND METHODS}

The study sample calculations based on previous research work concerned with similar study purpose suggested that, in order to reach firm conclusions, we should study 52 subjects. For the determination of the sample size, a total of 354 children were examined, of whom 52 fulfilled the inclusion criteria. All the patients examined in this study were obtained from Pediatric Dentistry Department, Faculty of Dentistry, Cairo University. All parents or guardians of participants were informed about the purpose of research and treatment procedures.
However, they were willing to overweigh the effects. Ethical approval for the study was granted by the Research Ethics Committee of Faculty of Dentistry, Cairo University. All participants met the following inclusion criteria:

1) Signed consent form;

2) Age group of patients between 7 years and 9 years;

3) Having unilateral mandibular first and/or second primary molars with interproximal caries (damaged side) and the contralateral teeth (intact side) having an clinically intact proximal contacts (served as control);

4) Having all their mandibular incisors erupted;

5) Having all their mandibular first permanent molars erupted in occlusion and clinically sound, and

6) No other missing teeth resulting from extraction or trauma;

Impressions for mandibular arch of the subjects were taken using irreversible hydrocolloid impression material (Zhermack alginate impression material, Badia Polesine (Rovigo) - Italy) with perforated metal trays. They were immediately poured in super hard dental stone plaster according to the manufacturing instructions to avoid any distortion, then for every dental cast a proper plaster base was made and trimmed then labeled with certain number. Space change was defined as the distance between the midpoint on the mesial surface of the mandibular permanent first molar and the midpoint on the distal surface of the mandibular primary canine (D E Space). Each of these landmarks was marked on the study casts by a sharp lead pencil to facilitate accurate recognition. The intact contralateral side was served as control. All the variables were measured using a digital caliper (Ayni, Micron Digital Calipers, China) with an accuracy of $0.01 \mathrm{~mm}$. 


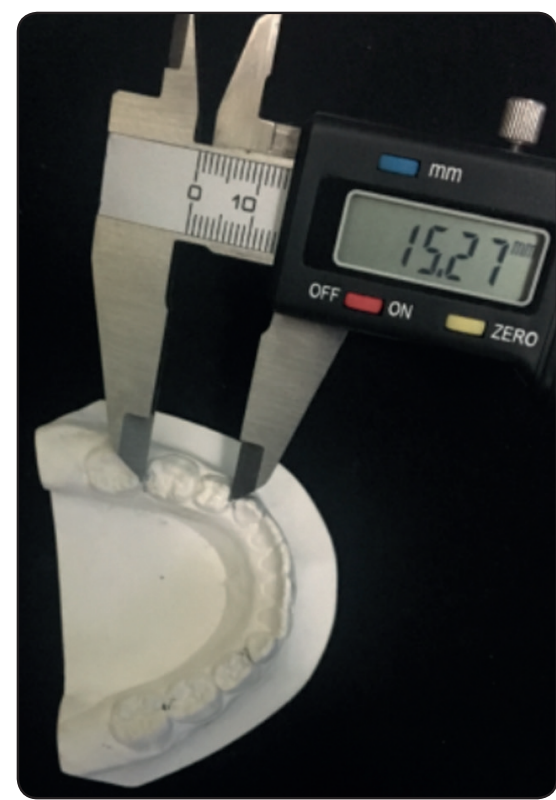

Fig. (1) Measuring DE space using digital caliper

To minimize the possibility of measurement errors, all measurements were repeated two times by the same examiner on separate occasions from which, an average value of the measurement in millimeter was recorded and analyzed. To minimize the possibility of bias, inter examiner reliability was assessed as the measurements were performed by two examiners according to the method described by Hunter and Priest, $1960{ }^{[7]}$.

\section{Statistical Analysis}

\section{Sample size calculation}

This study was planned to clinically evaluate dental arch space change caused by interproximal caries of deciduous molars in a group of Egyptian children. A total sample size of 52 samples will be sufficient to detect large effect size $(\mathrm{f}=0.632)$ with power $95 \%$ and significance level $5 \%$.

\section{For the inter-examiner reliability test}

Cronbach's alpha reliability coefficient test ${ }^{[8]}$ was used to compare the consistency and reliability between the inter-examiner groups. Cronbach's alpha reliability coefficient test normally ranged between 0 and 1 . The closer the Cronbach's alpha coefficient to 1.0, the higher the reliability. An alpha of 0.8 is probably a reasonable goal. Statistically significant difference as $\mathrm{P}<0.05$ was defined.

\section{For data measurement}

Data were statistically described in terms of mean, standard deviation $( \pm \mathrm{SD})$, frequency $(\mathrm{n})$ and percentage (\%) when appropriate. Student $t$ tests was applied to test the significance difference between the means of the carious and non carious sides. The significance level was set at $\mathrm{p} \leq 0.05$. Statistical analysis was performed with IBM ${ }^{\circledR}$ SPSS ${ }^{\circledR}$ (SPSS Inc., IBM Corporation, NY, USA) Statistics Version 22 for Windows.

\section{RESULTS}

This study was conducted to assess the changes taking place in dental arch dimension (D E Space) caused by untreated interproximal caries of deciduous molars. Regarding D E space, the measurements were represented by distance between the midpoint on the mesial surface of the mandibular permanent first molar and the midpoint on the distal surface of the mandibular primary canine. In the present study, at least one mandibular primary molar was affected by interproximal caries in a total of 52 patients: $33(63.5 \%)$ in boys and 19 $(36.5 \%)$ in girls and $37(71.2 \%)$ of them showed both primary molars in the same quadrant were affected. The mean age of the patients was 8.3 years (range 7 to 9 years)

The amount of D E space on the damage sides was significantly reduced when compared to the control sides $(\mathrm{P}<0.001)$. The average space loss was amounted to $0.82( \pm 0.64) \mathrm{mm}$. (Table 1)

Cronbach's alpha results for inter-examiner reliability was greater than $0.900(0.943)$ which denoted high consistency and reliability of the measurements. 
TABLE (1) Mean, standard deviation (SD), minimum, maximum and mean changes of D E space for intact and damaged sides

\begin{tabular}{|c|c|c|c|c|c|}
\hline \multirow{5}{*}{ 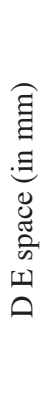 } & Mean & $\begin{array}{l}\text { intact } \\
\text { side }\end{array}$ & $\begin{array}{c}\text { Damaged } \\
\text { side }\end{array}$ & Change & $p$-value \\
\hline & Mean & 16.03 & 15.21 & 0.82 & \multirow{4}{*}{$0.001 *$} \\
\hline & SD & 1.07 & 0.83 & 0.64 & \\
\hline & Minimum & 15.42 & 15.09 & 0.37 & \\
\hline & Maximum & 16.67 & 16.13 & 0.93 & \\
\hline
\end{tabular}

* = significant

\section{DISCUSSION}

Despite recent advances in dental care that have increased the number of children appearing with intact dentition, caries of deciduous teeth still remains a problem. The loss of contact between the primary molars due to extensive carious involvement allows mesial movement of the permanent first molar and jeopardizes the arch length that is required for permanent dentition eruption. The loss of contact is an important factor in the etiology of a crowded arch at maturation. The presented study attempted to describe the amount of the space lost due to interproximal caries of first and second deciduous molars in mixed dentition stage.

The hypothesis that caries increases malocclusion rate is one that is difficult to evaluate. It has already been demonstrated that certain types of malocclusions, especially crowding, can increase caries rate, but it is not necessarily the case that the converse is true. Upon reviewing the literatures, many statements have been made regarding the effect of premature loss of primary molars on arch length deficiency. Up to our Knowledge, the D E space has never been assessed concerning the loss of proximal contacts due to interproximal caries. The theory is that caries, particularly interproximal caries, lead to a loss of arch space caused by mesial drift of adjacent teeth which increases malocclusion rate. This is similar to the malocclusions caused by premature extraction of primary teeth ${ }^{[9]}$. This mesial drift can lead to the creation of an Angle Class II or III dentition even in a person with a Class I skeletal pattern. Asymmetric caries can cause asymmetric mesial drift, creating situations where a person has a midline asymmetry or a situation where a person has an class I Angle classification of on one side, but a classification of II or III on the opposite side.

While most studies evaluate the relationship between premature loss of primary teeth and malocclusion, very few studies evaluate the relationship between interproximal tooth structure lost due to caries and malocclusion. One such study was performed by Northway and Wainright ${ }^{[10]}$, they found that in the early mixed dentition, crowding was more prevalent in subjects with Interproximal caries . They also found that asymmetric Angle classification was more prevalent in subjects with interproximal caries, while symmetric Angle classification was more prevalent in subjects with intact proximal contacts. Since this is the only study in the literature that evaluates the relationship between interproximal caries and malocclusion, it is difficult to draw definitive conclusions on the relationship between loss of proximal contacts due to caries and malocclusion ${ }^{[10]}$

In agreement with previous study Ben-Bassat et al. ${ }^{[1]}$ our results also found a significant loss of D E space of the damaged sides when compared to the intact sides. We found an average space loss of $0.82 \pm 0.64 \mathrm{~mm}$ in the distance between the midpoint on the mesial surface of the mandibular permanent first molar and the midpoint on the distal surface of the mandibular primary canine ( D E space) when compared to the control side in the lower jaw.

The timing of intervention requires close observation of the developing dentition since substantial loss of arch length cannot be easily 
recovered. In an overall sense, the critical nature of adequate arch length in every patient requires that the practitioner must use an appropriate modality to protect the developing dentition during the transitional phase of oral maturation when the position of the first permanent molar must be protected.

\section{CONCLUSION}

The amount of space needed for eruption of permanent teeth decrease significantly after loss of proximal contacts of primary molars due to interproximal caries in mixed dentition stage.

\section{Limitation}

This study was also limited because it was crosssectional. While subjects in the early mixed dentition could be compared to subjects in the late mixed dentition, the subjects themselves are different. A longitudinal study would eliminate this limitation, but since a longitudinal study would require that a population of children with high caries rates receive no treatment for their caries, it is inherently unethical and is therefore unlikely to be performed.

One important limitation of this study was the challenge to find the cases of unilateral interproximal caries, as it is generally recognized that the occurrence of dental caries in teeth are symmetrical and bilaterally equal.

\section{Recommendation}

It is the responsibility of the deciduous dental care physician to monitor the developing dentition in children. To facilitate the transition from deciduous to permanent dentition children should visit the dentist regularly to avoid complications of dental caries in primary teeth.

\section{REFERENCES}

1- Rao AK, Sarkar S. Changes in the arch length following premature loss of deciduous molars. J Indian Soc Pedod Prev Dent 1999; 17(1): 28-32.

2- Barberia E \& Lucavechi T. Free end Space Maintainers: Design Utilisation and Advantages. J Clin Pediatr Dent.; Fall 2006; 31(1):5-8.

3- Vikas S, Inder K P, Nikhil S, Neeraj G and Harveen KS.Space maintainers in Dentistry: Past to Present. J Clin Diagn Res. Oct 2013; 7(10):2402-2405.

4- $\quad$ Rao AK \& Sarkar S.Changes in arch length following premature loss of deciduous molars. J Indian Soc Pedo Prev Dent. 1999; March: 28-32.

5- $\quad$ Seward FS. Natural closure of the deciduous molar extraction spaces. Angle Orthodontist 1965; 35: 85-94.

6- Northway WM, Wainright RL, Demirjian A. Effects of premature loss of deciduous molars. Part I. Changes in posterior arch dimensions. Part II. Source of space change. Part III. Age at exfoliation and its influence on rate of space change. Part V. Role of occlusion in tooth migration. Part VI. Models of space closure. Angle Orthodontist 1984; 54: 295-29.

7- Hunter WS, Priest WR. Errors and discrepancies in measurements of tooth size. J dent Res. 1960. 39:405-414.

8- Kameron A, Dermaut L, Verbeeck R. The clinical significance of error measurement in the interpretation of treatment results.Eur J Orthod 2001. 23; 569-578.

9- Proffit, W. R., Fields, H. W., and Sarver, D. M.: Contemporary Orthodontics 5th ed. St. Louis, MO, Mosby, 2013.

10- Northway MW, Wainright RW. D E space--a realistic measure of changes in arch morphology: space loss due to unattended caries.J Dent Res.1980 Oct;59(10): 1577-80.

11- Ben-Bassat, Y., Harari, D., and Brin, I.: Occlusal traits in a group of school children in an isolated society in Jerusalem. Br. J. Orthod. 1997. 3;229-235. 